\section{Increasing awareness of eGFR monitoring}

We are grateful to the Journal for highlighting the important issue of epidermal growth factor receptor (eGFR) monitoring in psychiatric patients prescribed lithium. ${ }^{1}$ We recently carried out an audit of renal function monitoring across Camden and Islington NHS Foundation Trust. The aim was to assess whether renal investigations for grade 3 chronic kidney disease and referrals for specialist advice are being documented according to the 2006 Royal Colleges of Physicians and General Practitioners and Renal Association guidance ${ }^{2}$ in the notes of those psychiatric in-patients currently prescribed lithium across the Trust. These guidelines were recommended to psychiatrists in October 2007 (www.rcpsych.ac.uk/ members/rcpsychnews/october2007.aspx). Currently, eGFR is not part of the Trust-wide lithium blood-monitoring documentation.

A total of 303 sets of in-patients' notes were reviewed from across the Trust. An audit tool was designed to record patient information relating to the lithium regime and serial recording of eGFR. It was also recorded whether investigative parameters were documented to have been carried out in the presence of abnormal eGFR results. Requests for specialist medical opinions were also noted. Electronic pathology results were used where there was no written record in patient notes.

Of 18 in-patients prescribed lithium:

(a) $3(16.7 \%)$ patients had one-off abnormal results with other values recorded within the normal range;

(b) $1(5.6 \%)$ patient had one documented eGFR (54) that was abnormal. Owing to their African-Caribbean ethnicity, this may not have been significant;

(c) $3(16.7 \%)$ patients had no eGFR results recorded;

(d) no patients had eGFR documented in their notes.

When eGFR was abnormal, no further investigations were documented or specialist opinions sought.

This audit demonstrates that eGFR is not routinely monitored or documented in patients in our Trust who are on lithium, despite the guidance.
No outcome was documented on abnormal values. When values were abnormal, further investigations were not documented to have been performed. This supports Morriss \& Benjamin's ${ }^{1}$ view that psychiatrists require education about recent developments in renal monitoring in patients on lithium.

The audit results were presented at a local educational meeting and various local recommendations were made with the Pharmacy Department to improve awareness and practice.

There was agreement that the current lithium documentation charts should be modified to include eGFR as a routine part of the lithium work up and ongoing monitoring process. In conjunction with the Pharmacy Department, a brief, tabular form of the College guidance was developed to be incorporated onto Trust lithium-monitoring forms and in-patient pathology result forms. It was suggested that the Medical Education Department would add the guidance to the pharmacy section of the junior doctors' induction training package. The importance of documenting patients' ethnicity, age and gender on blood request forms in order that eGFR can be accurately calculated was also highlighted.

It is hoped that these recommendations may be helpful in assisting other mental health organisations both in monitoring their own practice, and in raising awareness among clinicians and other staff of the importance of eGFR monitoring in patients prescribed lithium therapy in hospital.

1 Morriss R, Benjamin B. Lithium and eGFR: a new routinely available tool for the prevention of chronic kidney disease. Br J Psychiatry 2008; 193: 93-5.

2 Royal College of Physicians, Renal Association. Chronic Kidney Disease in Adults. UK Guidelines for Identification, Management and Referral, 2006. Royal Colleges of Physicians and General Practitioners and Renal Association.

Vivienne S. Gould, Highgate Mental Health Unit, K Block, Dartmouth Park Hill, London N19 5NX. Email: vivgreen@talktalk.net; Mahnaz Hashmi, Highgate Mental Health Unit, London; Nikul Amin, Anisha Doshi, Royal Free and University College Medical School, London, UK

doi: 10.1192/bjp.194.2.191

\title{
Corrections
}

${ }^{*}$ Cover picture: Norris Embry 1921-1981. BJP, 193, A21. Text was written by Dr Alexandra Pitman; edited by Allan Beveridge.

${ }^{\star}$ Neuropsychiatric systemic lupus erythematosus associated with neuroleptic malignant syndrome. BJP, 193, 507-508. Authorship should read: Philippe Verdoot, MD, Eric L. Constant, MD, $\mathrm{PhD}$ and Arlette Seghers, MD, Université Catholique de Louvain, Cliniques Universitaires Saint Luc, Adult Psychiatric Unit, Belgium. Email: philippe.verdoot@uclouvain.be.

*The online Journal has been corrected post-publication in deviation from print and in accordance with these corrections.
Early intervention for adolescents with borderline personality disorder using cognitive analytic therapy: randomised controlled trial. BJP, 193, 477-484. In the summary, the first sentence of Conclusions should read: Both CAT and GCC are effective in reducing externalising psychopathology in teenagers with subsyndromal or full-syndrome borderline personality disorder.

Repetition of acute poisoning in Oslo: 1-year prospective study. $B J P, 194,73-79$. The fourth author's qualifications should read: Knut Erik Hovda, MD, PhD. 